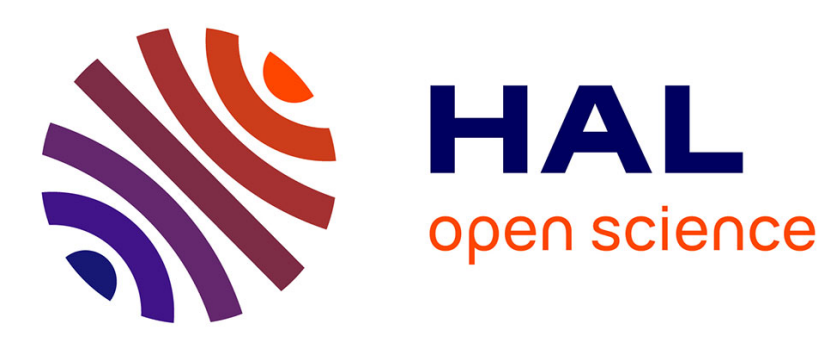

\title{
Stable Distributions of Growing and Coalescing Droplets
}

Bernard Derrida, C. Godrèche, I. Yekutieli

\section{To cite this version:}

Bernard Derrida, C. Godrèche, I. Yekutieli. Stable Distributions of Growing and Coalescing Droplets. EPL - Europhysics Letters, 1990, 12 (5), pp.385-390. 10.1209/0295-5075/12/5/001 . hal-03285597

\section{HAL Id: hal-03285597 \\ https://hal.science/hal-03285597}

Submitted on 21 Jul 2021

HAL is a multi-disciplinary open access archive for the deposit and dissemination of scientific research documents, whether they are published or not. The documents may come from teaching and research institutions in France or abroad, or from public or private research centers.
L'archive ouverte pluridisciplinaire HAL, est destinée au dépôt et à la diffusion de documents scientifiques de niveau recherche, publiés ou non, émanant des établissements d'enseignement et de recherche français ou étrangers, des laboratoires publics ou privés. 
Europhys. Lett., 12 (5), pp. 385-390 (1990)

\title{
Stable Distributions of Growing and Coalescing Droplets.
}

\author{
B. Derrida (*), C. GodrÈche (**) and I. Yekutieli (**) \\ (*) Service de Physique Théorique CEA, Saclay - 91191 Gif-sur-Yvette Cedex, France \\ (**) Service de Physique du Solide et de Résonance Magnétique, CEA, Saclay \\ 91191 Gif-sur-Yvette Cedex, France
}

(received 16 March 1990; accepted in final form 4 May 1990)

PACS. 02.50 - Probability, theory, stochastic processes, and statistics.

PACS. 05.20 - Statistical mechanics.

PACS. $64.70 \mathrm{~F}$ - Liquid-vapour transitions.

PACS. 68.45 - Solid-fluid interface processes.

\begin{abstract}
We consider two simplified models of the formation of patterns emerging from the growth and coalescence of water droplets on a substrate (breath figures). The study is restricted here to the case of a one-dimensional substrate. In the first model we assume a monodisperse distribution of droplet sizes. In the second model, obtained as a mean-field approximation of the first one, the distribution of distances between neighbouring droplets obeys a Smoluchowski equation. We solve this equation analytically to obtain the coverage of the line (fraction of it covered by droplets) and the distribution of distances between droplets. We conclude by discussing the relevance of the random parking problem for breath figures.
\end{abstract}

Introduction. - The problem of the combined effects of the growth and coalescence of water droplets condensing on glass surfaces has motivated several recent works, both on experimental and theoretical aspects [1-7]. This problem is the laboratory version of the well-known phenomenon of condensation of vapour on spectacles or dew on grass. Experimental studies of breath figures, i.e. the patterns formed by the droplets, performed on two- and one-dimensional substrates $[1,6,7]$, show the following features: after a transient regime where the droplets grow individually, coalescences between droplets appear. The coverage of the substrate (the fraction of it occupied by droplets) reaches a constant value in time. In this stage the breath figures are self-similar, i.e., up to a rescaling of lengths, the figures look similar at successive times; the values of the 1- or 2-dimensional coverages are close to those of the random parking problem [8-10] in the same dimensions. In a third regime, new droplets nucleate and grow in the space left between the original droplets.

Inspired by these experiments, and discarding the last regime, the following model has been proposed $[2,3,5,7]$. At time $t=0$, nucleation sites are randomly distributed on a substrate of dimension $d$ ( $d=2$ corresponds to droplets on a plate of glass, $d=1$ on a spider's web). On these sites, droplets of dimensionality $D$ grow ( $D=3$ for real droplets); the intrinsic growth (i.e. between two coalescences) of the radius of a droplet follows some 
power law in time. When a coalescence between two droplets (of radii $R_{1}$ and $R_{2}$ ) occurs, a new droplet forms at the centre of mass of these two droplets, with a radius $R$ given by mass conservation

$$
R^{D}=R_{1}^{D}+R_{2}^{D} .
$$

Numerical simulations have been performed for this model [3-7]. They reproduce the experimental observations mentioned above, in particular the existence of a scaling regime at large times. However a detailed analytical treatment of this problem has not yet been proposed.

The purpose of the present work is to introduce two simplified $1-d$ versions (models $A$ and $B$ ) of the above problem in which the polydispersity in size of the droplets has been eliminated. These models capture the essence of the physical phenomenon, i.e. the existence of a scaling regime which, at least for the second model, can be described by an analytical approach.

Model $A$. - We first consider a system of equally sized droplets on a line (1- $d$ substrate). The radius $R$ of these droplets grows in time. The precise form of this growth law is irrelevant in this model, as will become clear below. When two neighbouring droplets coalesce, a new droplet is created with the same radius $R$, centred half-way between the coalescing droplets. This model can be formally viewed as a limit of the model of $D$ dimensional droplets on a 1- $d$ substrate, described in the introduction, when $D \rightarrow \infty$. Since at all times the radii of the droplets are all equal, the problem is reduced to studying the distribution of distances (or bonds) $h$ between the centres of neighbouring droplets. When the radius of the droplets reaches a certain value $R$, all the pairs of neighbouring droplets which are at a distance $h_{0}(=2 R)$ coalesce and all the remaining distances $h$ are larger than $h_{0}$. Therefore if the system is represented by a sequence of points along the line (which are the centres of the droplets), the dynamics of growth and coalescence reduces to searching the minimal distance $h_{0}$ between two adjacent points, and replacing these two points by one point midway between them. Hence the two bonds neighbouring the disappearing bond $h_{0}$ become longer by $h_{0} / 2$.

If one starts at time $t=0$ with a system of $N$ bonds, and if we denote by $N g_{t}(h) \delta h$ the number of bonds of lengths between $h$ and $h+\delta h$ at time $t$ (at which time, the length of the minimal bond is $h_{0}$ ), one can define the fraction $f_{t}(h)$ of bonds of length $h$ by

$$
f_{t}(h)=g_{t}(h)\left[\int_{h_{0}}^{\infty} g_{t}\left(h^{\prime}\right) \mathrm{d} h^{\prime}\right]^{-1} .
$$

The length $h_{0}$ of the minimal bond is a natural time scale in the problem. It is therefore convenient to rescale the distribution $f_{t}(h)$, defining the distribution $F(x, t)$ of the reduced variable $x=h / h_{0}$ :

$$
f_{t}(h)=h_{0}^{-1} F\left(h / h_{0}, t\right) .
$$

The coverage $C_{t}$ of the line at time $t$ is then given by

$$
C_{t}=h_{0}\left[\int_{h_{0}}^{\infty} h f_{t}(h) \mathrm{d} h\right]^{-1}=\left[\int_{1}^{\infty} x F(x, t) \mathrm{d} x\right]^{-1},
$$

using the fact that the number of droplets (all of diameter $h_{0}$ ) is equal to the number of bonds. 
Figure 1 shows $F(x, t)$, obtained by numerical simulations of model $A$, at 3 different times, namely when the number of remaining bonds are 100000,50000 and 25000 , starting with a system of 400000 bonds randomly distributed between 0 and 1 . These three curves are superimposable, giving evidence to scaling at large times. It is also found that for large times, $C_{t} \simeq 0.72$, to be compared to $C_{t} \simeq 0.8$ found in 1- $d$ experiments and simulations [7].

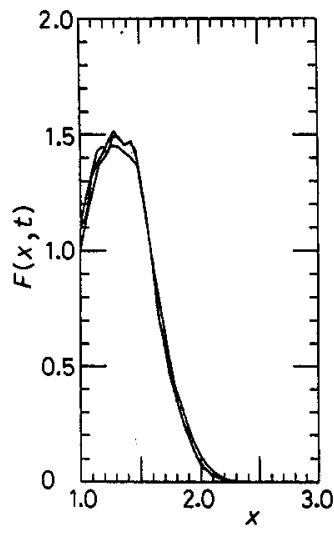

Fig. 1.

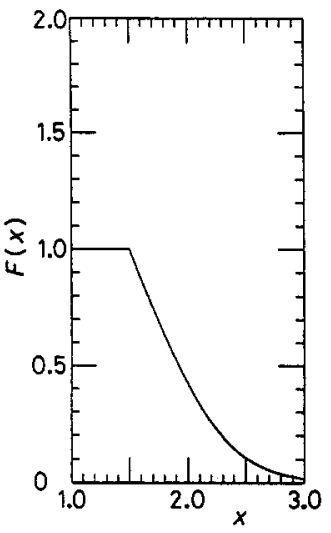

Fig. 2.

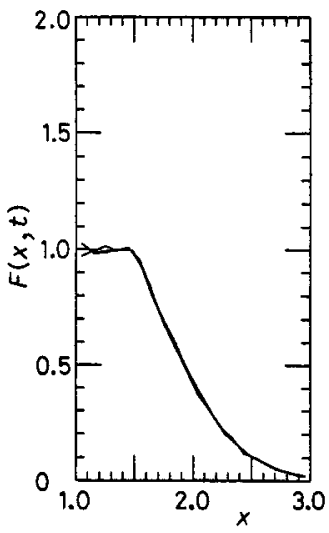

Fig. 3.

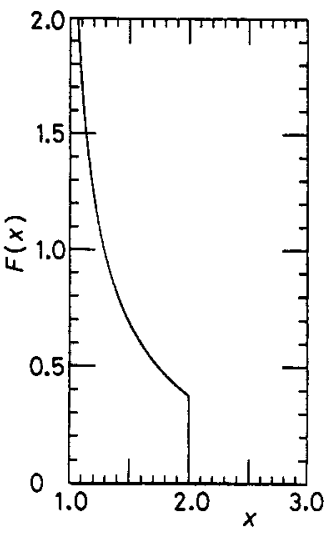

Fig. 4.

Fig. 1. - The scaled distribution $F(x, t)$ obtained by simulation of model $A$ at 3 different times.

Fig. 2. - The scaled stationary distribution $F(x)$ for model $B$ obtained by iteration of eq. (6).

Fig. 3. - Same as fig. 1, for model $B$.

Fig. 4. - The distribution of distances between the centres of adjacent cars for the random parking problem.

Model B. - This model is a mean-field approximation of model $A$ : the correlations between bonds are neglected. Hence, at each time step, the shortest bond $h_{0}$ is cut into two pieces of equal length and the pieces are added to two randomly chosen bonds. If $N g_{t}(h) \delta h$ is the numer of bonds of length between $h$ and $h+\delta h$ at time $t$, and if during the time interval $(t, t+\delta t)$ all bonds of length between $h_{0}$ and $h_{0}+\delta h$ are removed, one can write the following equation for $g_{t}(h)$ :

$$
g_{t+\delta t}(h)=g_{t}(h)+2 g_{t}\left(h_{0}\right) \delta h\left[g_{t}\left(h-\frac{1}{2} h_{0}\right) \theta\left(h-\frac{3}{2} h_{0}\right)-g_{t}(h)\right]\left[\int_{h_{0}}^{\infty} g_{t}\left(h^{\prime}\right) \mathrm{d} h^{\prime}\right]^{-1} .
$$

The two terms inside the brackets of the r.h.s. of eq. (5) correspond to the gains and the losses in this Smoluchowski equation; the Heaviside function accounts for the fact that no bonds are smaller than $h_{0}$. If we introduce, as in model $A$, the fraction $f_{t}(h)$ of bonds of length $h$ and the rescaled distribution $F(x, t)$ (see eqs. (2), (3)), we find from eq. (5) (using the fact that $\delta h$ and $\delta t$ are small and that $\left.\int g_{t+\delta t}(h) \mathrm{d} h=\int g_{t}(h) \mathrm{d} h-g_{t}\left(h_{0}\right) \delta h\right)$ that $f_{t}(h)$ and $F(x, t)$ satisfy

$$
\begin{gathered}
f_{t+o t}(h)=f_{t}(h)+f_{t}\left(h_{0}\right) \delta h\left[2 f_{t}\left(h-\frac{1}{2} h_{0}\right) \theta\left(h-\frac{3}{2} h_{0}\right)-f_{t}(h)\right], \\
\gamma(t) \frac{\partial F(x, t)}{\partial t}=F(x, t)+x \frac{\partial F(x, t)}{\partial x}+F(1, t)\left[2 F\left(x-\frac{1}{2}, t\right) \theta\left(x-\frac{3}{2}\right)-F(x, t)\right],
\end{gathered}
$$


where $\gamma(t)=h_{0} \delta t / \delta h$ represents the relation between the real time $t$ and the minimal length $h_{0}$. Since this relation is arbitrary in this model, we will choose for simplicity $\gamma(t)=1$. The whole time dependence of the process is reduced to solving eq. (7) with some initial condition $F(x, 0)$, or, introducing the Laplace transform $\phi(p, t)$ of $F(x, t)$

$$
\phi(p, t)=\int_{1}^{\infty} F(x, t) \exp [-p x] \mathrm{d} x
$$

to solving the differential equation

$$
\frac{\partial \phi(p, t)}{\partial t}=F(1, t)[2 \exp [-p / 2]-1] \phi(p, t)-F(1, t) \exp [-p]-p \frac{\partial \phi(p, t)}{\partial p} .
$$

Though we did not find a way of solving analytically eq. (7) or eq. (9) for arbitrary initial conditions, all the stationary solutions $\phi(p)$ of eq. (9) may be obtained, since they satisfy an ordinary differential equation. These solutions are

$$
\phi(p)=F(1) \int_{p}^{\infty} \frac{\mathrm{d} t}{t} \exp \left[-t+F(1) \int_{p}^{t} \mathrm{~d} u \frac{1-2 \exp (-u / 2)}{u}\right] \text {, }
$$

indexed by the continuous parameter $F(1)$. The question is what stationary solution is reached for given initial conditions $\phi(p, 0)$ or $F(x, 0)$, if any at all. We can argue that the stationary solution which is obtained for most initial conditions $F(x, 0)$ satisfies

$$
F(1)=1 \text {. }
$$

The argument is as follows. From eq. (10) one can show that for $p$ small

$$
1-\phi(p) \sim p^{F(1)} .
$$

So since the first moment of $x$ is given by

$$
\bar{x}=\int_{1}^{\infty} x F(x) \mathrm{d} x=-\phi^{\prime}(0)
$$

and noting that $\phi(0)=1$, the only stationary solution which gives a finite nonzero value of $\bar{x}$ satisfies $F(1)=1$. $F(1)<1$ corresponds to $\bar{x}=\infty$, whereas $F(1)>1$ corresponds to $\bar{x}=0$. This last case should be ruled out since $F(x)$ is positive and of support $[1, \infty]$.

One can distinguish between the two remaining cases for $F(1)$ by looking at the trajectories of the singularities of $\phi(p)[11]$. Assume that at time $t, \phi(p, t)$ has some powerlaw singularity in the complex plane of $p$ at $p_{i}(t)$

$$
\phi_{\text {sing }}(p, t) \sim\left(p-p_{i}(t)\right)^{\beta} .
$$

By equating the most singular terms of both sides of eq. (9), we get an equation of motion for the singularity $p_{i}(t)$

$$
\mathrm{d} p_{i}(t) / \mathrm{d} t=p_{i}(t)
$$


If the initial condition $F(x, 0)$ is such that $\phi(p, 0)$ has some singularities $p_{i}(0) \neq 0$ in the complex $p$ plane, these singularities will go to infinity when $t \rightarrow \infty$. One then expects $\phi(p, t)$ to converge to a stationary solution analytic in $p$, implying that $F(1)$ in eq. (12) has to be an integer. Moreover, since $0<F(1) \leqslant 1$, one finds that $F(1)=1$.

If the initial condition $F(x, 0)$ decays slowly when $x \rightarrow \infty$, so that $\phi(p, 0)$ is singular at $p=0$, then the singularity of $\phi(p, t)$ might remain at $p=0$ and $F(x, t)$ might converge to another stationary solution with $F(1)<1$.

Using eqs. (10), (11), (13) one can calculate $\bar{x}$

$$
\bar{x}=\int_{0}^{\infty} \frac{\mathrm{d} t}{t^{2}}\left[1-\exp \left[-t+2 \int_{0}^{t} \frac{1-\exp [-u / 2]}{u} \mathrm{~d} u\right]\right] 1.547
$$

and deduce from eq. (4) the coverage $C=1 / \bar{x} \simeq 0.64$ in this mean-field approximation.

In fig. 2 and 3 we compare the stationary distribution obtained by iterating eq. (6) for $F(1)=1$ (fig. 2), and the results of a simulation of model $B$ (fig. 3). We see that the agreement is quite satisfactory.

As a final remark, let us mention that other cases of scaling solutions are known for Smoluchowski equations $[12,13]$.

Conclusion. - We have performed simulations of the model presented in the introduction with $D$-dimensional droplets on a line. For all $D \geqslant 2$, after a transient regime, the patterns emerging are self-similar in time: the distributions of droplet sizes and inter-droplet distances obey simple scaling relations. These distributions resemble, as $D \rightarrow \infty$, the distributions found for model $A$. We will present these results in a future work.

Breath figures display intriguing similarities with the random parking problem. In 1-d this is the problem of sequentially parking cars of length 1 at random along an infinitely long street, until the street is "jammed": the spaces between the cars are all smaller than 1. As mentioned in the introduction, the final density of cars along the street is close to the coverage found in breath figures experiments and simulations on 1- $d$ substrates. The same is found for the 2-d versions of these problems. All the more, in 1- $d$, breath figures in the selfsimilar regime are in nearly-jammed states. This is seen in fig. 1, where almost all the weight of the distribution of bonds lies between $h_{0}$ and $2 h_{0}$, implying that almost all gaps between the droplets are smaller than $h_{0}$. Nevertheless, the analogy is not complete: comparing fig. 1 and 4, we see that the distributions of distances between droplet- and carcentres are very different; in the random parking problem (fig. 4) the distribution diverges (logarithmically) at 1 and drops discontinuously to 0 at 2 .

In this paper we have seen that a very simplified mean-field model for the formation of breath figures, solvable analytically, possesses, at least qualitatively, the main features (scaling regime) of more complicated models (the model described in the introduction and model $A$ ). Starting from this mean-field approach, one should be able to improve it by taking into account some correlations between the lengths of neighbouring bonds. Our first attempts in this direction are encouraging and we hope to present them in a forthcoming paper.

It is a pleasure to thank D. BEYSENS, P. GUÉNOUN and A. STEYER for introducing us to the subject and for many helpful discussions, and D. BESsis for an illuminating discussion on Laplace transforms. 


\section{REFERENCES}

[1] Beysens D. and KNobler C. M., Phys. Rev. Lett., 57 (1986) 1433 and references to earlier works therein.

[2] Viovy J. L., Beysens D, and Knobler C. M., Phys. Rev. A, 37 (1988) 4965.

[3] Fritter D., Knobler C. M., Roux D. and Beysens D., J. Stat. Phys., 52 (1988) 1447.

[4] FritTer D., Thesis, University of California, Los Angeles, Cal. (1989).

[5] Meakin P. and Family F., J. Phys. A, 22 (1989) 225.

[6] Briscoe B. J. and Galvin K. P., preprint (1989).

[7] Steyer A., Guénoun P., Beysens D., Fritter D. and KNobler C. M., submitted to Europhys. Lett.

[8] RÉnyi A., Publ. Math. Inst. Hung. Acad. Sci., 3 (1958) 109.

[9] Widom B., J. Chem. Phys., 44 (1966) 3888.

[10] Swendsen R., Phys. Rev. A, 24 (1981) 504 and other references therein.

[11] Thual O., Frisch U. and Hénon M., J. Phys. (Paris), 46 (1985) 1485.

[12] Ernst M. H., Fractals in Physics, edited by L. Pietronero and E. Tosatti (Elsevier, Houston, Tex.) 1986, p. 289.

[13] Van Dongen P. G. J. and ERnst M. H., J. Stat. Phys., 49 (1987) 879 and 927. 